\section{Spinal dural arteriovenous fistula: a treatable cause}

\section{of myelopathy}

The case: A previously healthy 46 year-old man, who works as a standing forklift operator, presented to an emergency department with a 1-day history of bilateral leg "soreness" and numbness in his right leg ascending to his groin. Seven-months before while lifting a heavy box, he had experienced sudden pain in his right buttock and leg that was treated with anti-inflammatory medications and rest. Subsequently, he began to experience bilateral leg fatigue with moderate activity, occasional nocturnal urinary incontinence, stiffness of gait

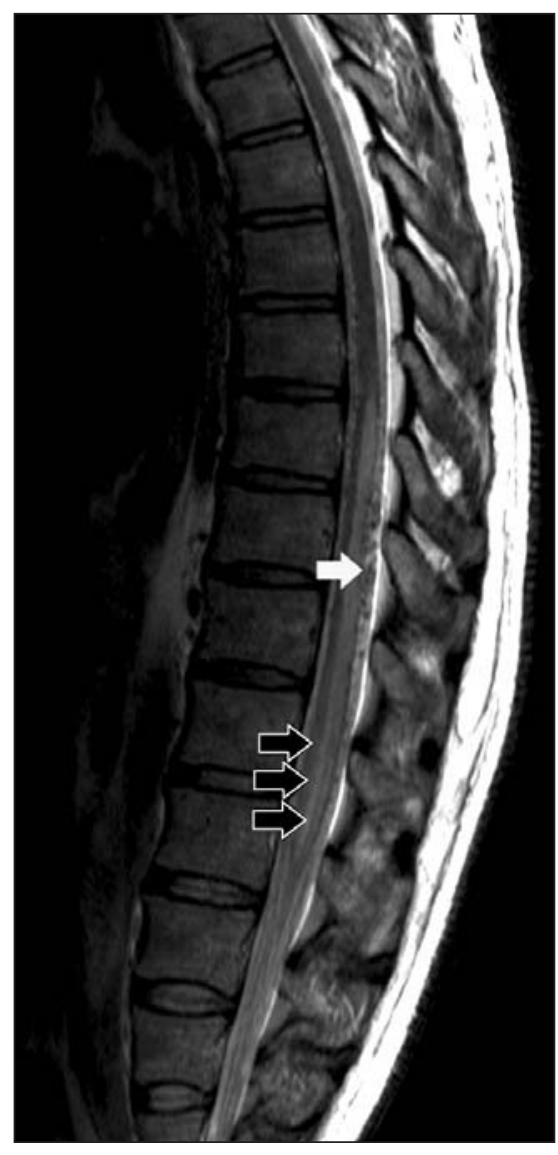

Figure 1: Sagittal $T_{2}$-weighted magnetic resonance image of the thoracolumbar spine showing edema of the thoracic cord and conus medullaris (black arrows) and regional dilated perimedullary vessels (white arrow) suggestive of a spinal vascular malformation. venous plexus (white arrow). with dragging of the right leg and leg weakness elicited by hot baths (Uhthoff phenomenon). The patient also reported a 12-month history of intermittent sharp left costal pain that followed a dermatomal distribution, from just below his left nipple to his mid back. The pain became worse with deep inspiration.

On physical examination, the patient was oriented and cooperative, and his vital signs were normal. The results of an examination of the cranial nerves and upper extremities were normal. He was able to stand, and he walked with a spastic gait and apprehension of falling. The patient had a normal range of spine movement with no tenderness to palpation over the length of the spine. A passive straight leg raise did not elicit pain. Muscle tone was increased in both of his lower extremities. Power in his iliopsoas, quadriceps and hamstrings was slightly weak, and he had normal power in his gastrocnemius, tibialis anterior and extensor halluces longus bilaterally. The patient's deep tendon reflexes were hyper-reflexic bilaterally in his lower extremities, his plantar reflex was downgoing bilaterally and clonus could not be elicited. The patient's rectal tone was normal and perianal sensation was intact, and his pinprick, light touch and vibration senses were intact in his lower extremities.

A plain and gadolinium-enhanced magnetic resonance image of the patient's whole spine showed intramedullary $T_{2}$-weighted hyperintensity and cord expansion from thoracic spinal cord at the T8 level to the conus medullaris with prominent perimedullary flow voids surrounding the spinal cord that were consistent with engorged vessels (Figure 1). A contrast

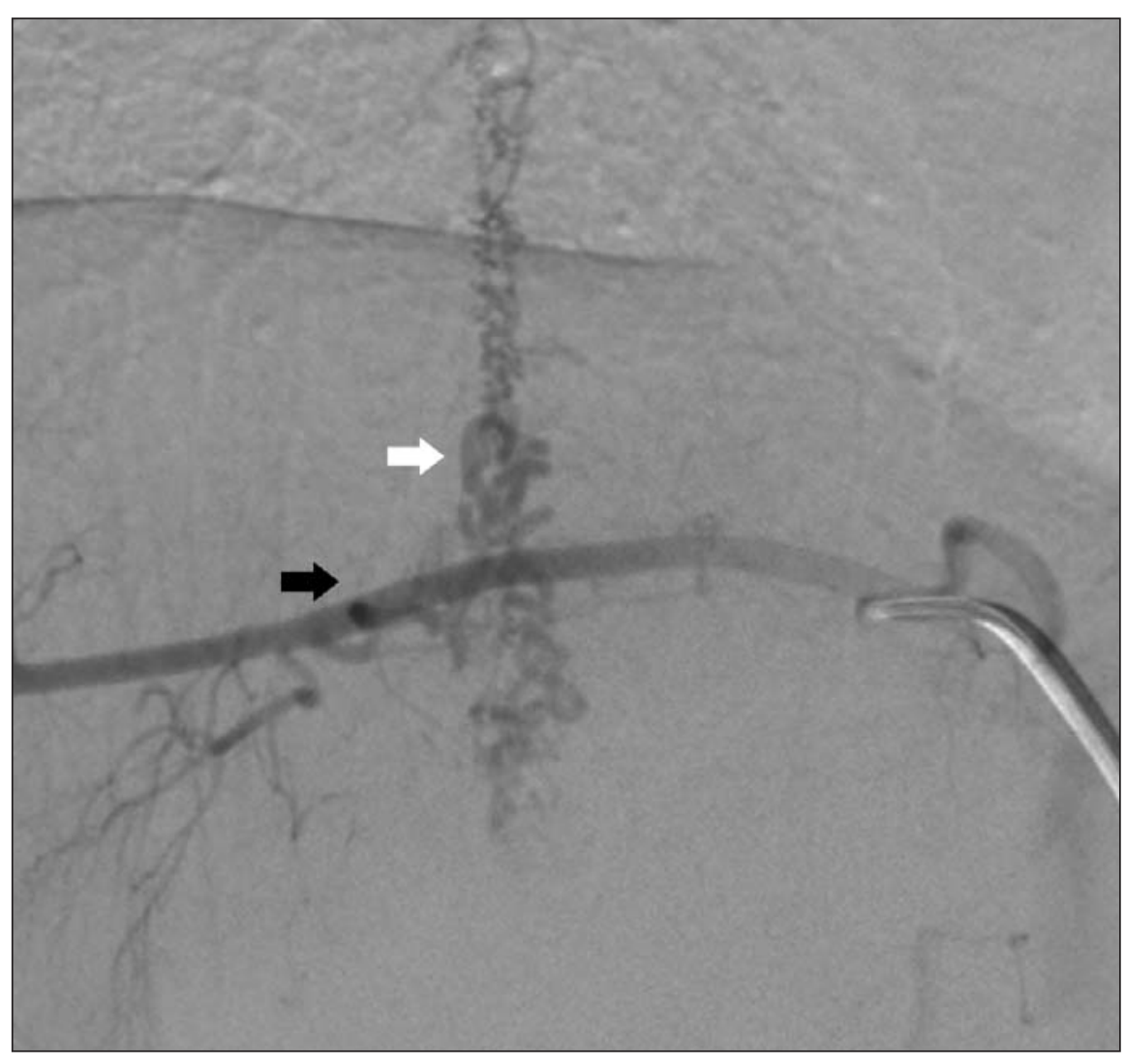

Figure 2: Selective spinal angiogram of the right T10 segmental artery showing a fistulous connection (black arrow) between the segmental artery and the perimedullary 


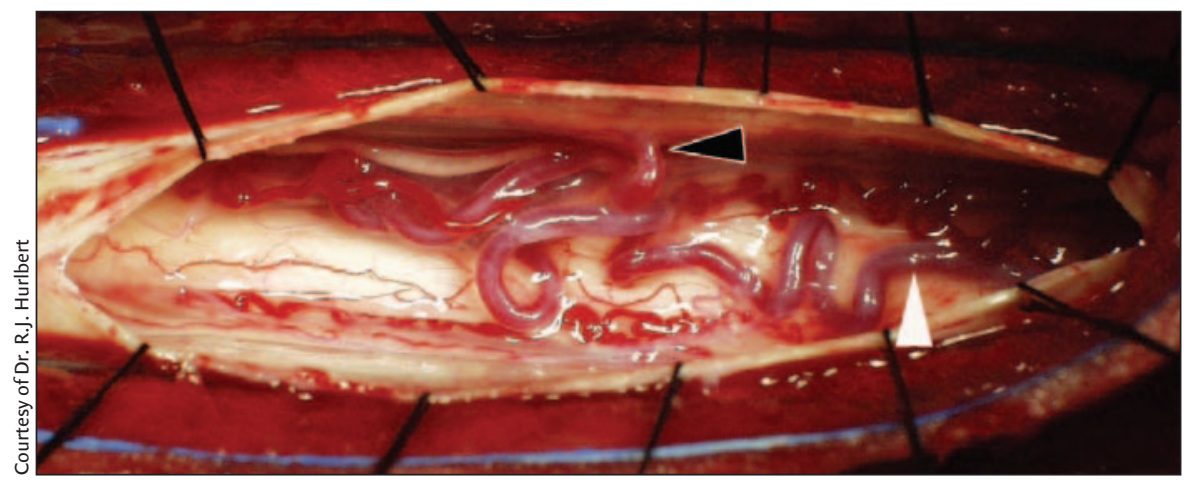

Figure 3: Intraoperative photograph of a spinal dural arteriovenous fistula (black arrow) and engorged perimedullary vessels (white arrow).

magnetic resonance angiogram of the thoracic and lumbar spine showed a feeding vessel at the right thoracic T10 level that was supplying an intraspinal vascular malformation. A catheter-based spinal angiogram confirmed the presence of a dorsal, dural arteriovenous fistula with extensive perimedullary venous reflux supplied from the right $\mathrm{T} 10 \mathrm{seg}$ mental artery (Figure 2).

The patient was operated on within 7 days of presentation because of progressive myelopathy. A posterior thoracic T9-T11 laminectomy and durotomy was performed, which showed engorged venous vessels on the surface of the spinal cord (Figure 3). An arterialized draining vein was identified arising from the inner aspect of the dural sleeve of the right T10 nerve root. This vein was cauterized and divided, which immediately reduced the turgor of the distended perimedullary venous plexus. Postoperatively, the patient regained full power in his legs with improvement in gait, and his pain was markedly reduced. He had mild urinary retention that was treated successfully with bethanechol chloride.

This patient presented with a history of work-related radicular leg pain as well as progressive weakness and signs and symptoms of myelopathy. The differential diagnosis for myelopathy is extensive; however, one starting point is the differentiation between structural and nonstructural lesions. Disorders that affect the spinal cord can be classified as being from bony, intervertebral diskrelated, tumorous, infectious, cystic or hemorrhagic causes. A thorough history should be obtained with emphasis on pain and neurologic symptoms, past trauma and risk factors for cancer and infection. A neurologic examination will help to determine whether a patient is affected by myelopathy (with upper motor neuron signs) or radiculopathy (with lower motor neuron signs) (Table I). Mixed upper and lower motor neuron findings may be present. A neurologic examination will also help to localize the lesion to the cervi$\mathrm{cal}$, thoracic or lumbar level. Once myelopathy is suspected, initial investigations should be guided by the patient's history and the results of a physical examination. However, urgent magnetic resonance imaging of the spinal column is almost always necessary for patients with myelopathy. In cases with a spinal dural ateriovenous fistula, the radiologic findings include regional $T_{2}$-weighted hyperintensity of the spinal cord and local dilated perimedullary vessels. ${ }^{1}$ Neurosurgical refer$\mathrm{ral}$ is appropriate if a structural lesion is identified.

The exact incidence of spinal dural ateriovenous fistula is unclear; however, our tertiary care centre, which serves a population of I.4 million, has about $\mathrm{I}-3$ cases each year. Spinal dural ateriovenous fistulas are 5 times more common among men compared with women, with a mean age at diagnosis of 55-60 years, and the most frequent location is the thoracolumbar spine. This condition arises from an acquired abnormal communication between a branch of a segmental spinal artery and a radicular vein connecting with the perimedullary venous plexus of the spinal cord. High venous pressure in the arterialized vessels is thought to produce edema and relative ischemia of the spinal cord, which results in myelopathic symptoms.

Symptoms of spinal dural ateriovenous fistula may be mistaken for more common spinal degenerative conditions such as lumbar spondylosis and neurogenic claudication, multiple sclerosis, spinal cord tumour, transverse myelitis and polyneuropathy. Delayed diagnosis is common, with the time from symptom onset to diagnosis ranging from $\mathrm{I2}$ to 36 months. ${ }^{1}$ Progressive weakness, muscle spasm, fecal incontinence, overflow urinary incontinence or urinary retention and erectile dysfunction are characteristic of myelopathy, but they

Table 1: Symptoms and signs of myelopathy and radiculopathy

\begin{tabular}{lll}
\hline Symptoms or signs & \multicolumn{1}{c}{ Myelopathy } & \multicolumn{1}{c}{ Radiculopathy } \\
\hline Symptoms & - Weakness of limbs, typically & - Weakness in muscles \\
proximal and bilateral & $\begin{array}{l}\text { supplied by affected nerve } \\
\text { roots }\end{array}$ \\
- Muscle spasm & - Dermatomal paresthesias \\
- Fecal incontinence & - Pain along dermatome \\
- Erectile dysfunction & \\
- Urinary retention or & \\
incontinence & \\
- Increased muscle tone & - Decreased muscle tone \\
- Hyper-reflexia & - Hypo-reflexia \\
& - Upgoing plantar reflex & - Downgoing plantar reflex \\
& - Clonus & - No clonus \\
\hline
\end{tabular}


are not specific to spinal dural ateriovenous fistula. These symptoms may be aggravated by activities that increase intra-abdominal pressure. In about onethird of cases, lower motor neuron findings precede upper motor neuron findings. ${ }^{1}$ Sensory findings may follow an ascending radicular pattern (Table I).

Spinal dural ateriovenous fistula has a variable course ranging from acute onset that mimics anterior spinal artery syndrome to chronic and progressive symptoms. ${ }^{1}$ Untreated, it may progress to subacute necrosis of the

Box1: Clinical features of spinal dural atriovenous fistula

- A rare but treatable cause of myelopathy

- May present with a mix of myelopathic and radicular findings

- Mean age at diagnosis ranges from 55 to 60

- More common in males spinal cord with permanent and severe impairment, including limb paralysis and loss of sphincter function (FoixAlajouanine syndrome).

Treatment options include open spinal surgery to disconnect the arterialized draining vein or endovascular embolization; however, surgery has a higher likelihood of cure. In contrast, the first choice treatment for other spinal arteriovenous malformations tends to be embolization. ${ }^{2}$ Recovery depends on the age of the patient and the severity of preoperative myelopathy. ${ }^{3}$ Most patients have improvement of their symptoms and a reversal of radiologic changes after treatment. ${ }^{4}$ Gait difficulty and muscle strength respond best to treatment, and micturition, pain and muscle spasms respond less well. ${ }^{1}$

Although it is a rare disease, clinicians should be aware of spinal dural arteriovenous fistula as a frequently misdiagnosed and progressively disabling neurologic condition that can be cured (Box I). Early clinical identification cou- pled with urgent imaging can shorten the time to diagnosis and treatment.

\section{Roberto Jose Diaz MD \\ John H. Wong MD MSc \\ Division of Neurosurgery \\ Department of Clinical Neurosciences \\ Foothills Medical Centre \\ University of Calgary \\ Calgary, Alta.}

This article has been peer reviewed.

Competing interests: None declared.

\section{REFERENCES:}

I. Jellema K, Tijssen CC, van Gijn J. Spinal dural arteriovenous fistulas: a congestive myelopathy that initially mimics a peripheral nerve disorder. Brain 2006;129:3150-64.

2. Veznedaroglu E, Nelson PK, Jabbour PM, et al. Endovascular treatment of spinal cord arteriovenous malformations. Neurosurgery 2006;59:S202-9.

3. Nagata S, Morioka T, Natori Y, et al. Factors that affect the surgical outcomes of spinal dural arteriovenous fistulas. Surg Neurol 2006;65:563-8.

4. Steinmetz MP, Chow MM, Krishnaney AA, et al. Outcome after the treatment of spinal dural arteriovenous fistulae: a contemporary single-institution series and meta-analysis. Neurosurgery 2004;55:77-87.

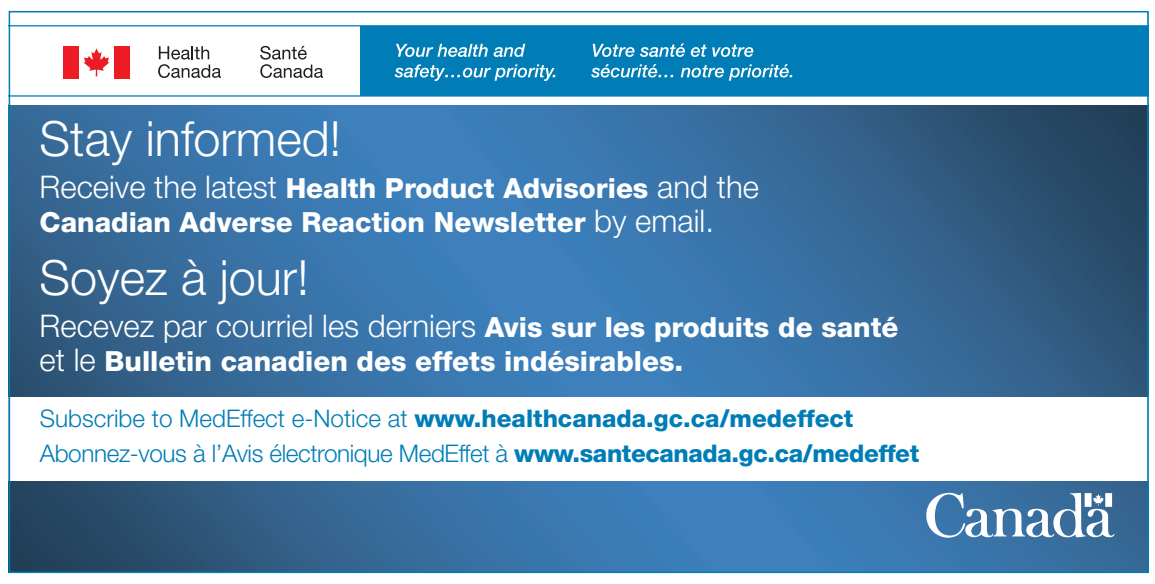

